\title{
UNIT AND UNITARY CAYLEY GRAPHS FOR THE RING OF EISENSTEIN INTEGERS MODULO $n$
}

\author{
Reza Jahani-Nezhad ${ }^{\dagger}, \quad$ Ali Bahrami ${ }^{\dagger \dagger}$ \\ Department of Pure Mathematics, Faculty of Mathematical Sciences, \\ University of Kashan, \\ Kashan 87317-53153, I. R. Iran \\ †jahanian@kashanu.ac.ir, ††alibahrami1972@gmail.com
}

\begin{abstract}
Let $E_{n}$ be the ring of Eisenstein integers modulo $n$. We denote by $G\left(E_{n}\right)$ and $G_{E_{n}}$, the unit graph and the unitary Cayley graph of $E_{n}$, respectively. In this paper, we obtain the value of the diameter, the girth, the clique number and the chromatic number of these graphs. We also prove that for each $n>1$, the graphs $G\left(E_{n}\right)$ and $G_{E_{n}}$ are Hamiltonian.
\end{abstract}

Keywords: Unit graph, Unitary Cayley graph, Eisenstein integers, Hamiltonian graph.

\section{Introduction}

Associating a graph with an algebraic object is an active research subject in algebraic graph theory, an area of mathematics in which methods of abstract algebra are employed in studying various graph invariants and tools in graph theory are used in studying various properties of the associated algebraic structure. The Cayley graph of a finite group was first considered in 1878 by Arthur Cayley [12]. Research on graphs associated with rings was started in 1988 by I. Beck [10]. In the literature, there are some other graphs associated with rings, such as the Cayley graph of a commutative ring [1], the unitary Cayley graph of a ring [3], the total graph of a ring [5], the zero divisor graph of a ring [6], the unit graph of a ring [7] and the comaximal graph of a ring [16].

Let $R$ be a commutative ring with non-zero identity. We denote by $U(R), J(R)$ and $Z(R)$ the group of units of $R$, the Jacobson radical of $R$ and the set of zero divisors of $R$, respectively. The unitary Cayley graph of a ring $R$, denoted by $G_{R}$, is the graph whose vertex set is $R$, and in which $\{a, b\}$ is an edge if and only if $a-b \in U(R)$. In 1995 this graph was initially introduced by Dejter and Giudici [14] for $\mathbb{Z}_{n}$, the ring of integers modulo $n$. In 2009, Akhtar et al. [3] generalized the unitary Cayley graph $G_{\mathbb{Z}_{n}}$ to $G_{R}$ for a finite ring $R$. The unit graph of a ring $R$, denoted by $G(R)$, is a graph whose vertices are elements of $R$ and two distinct vertices $a$ and $b$ are adjacent if and only if $a+b$ in $U(R)$. In 1990, the unit graph was first investigated by Chung [13] and Grimaldi [16] for $\mathbb{Z}_{n}$. In 2010, Ashrafi, et al. [7] generalized the unit graph $G\left(\mathbb{Z}_{n}\right)$ to $G(R)$ for an arbitrary ring $R$. Numerous results about unit and unitary Cayley graphs were obtained, see for examples $[3,7,18,19,21,22]$.

The following facts are well known, see for example [4] and [17]. Let $\omega$ be a primitive third root of unity. Then the set of all complex numbers $a+b \omega$, where $a$ and $b$ are integers, forms an Euclidean domain with the usual complex number operations and Euclidean norm $N(a+b \omega)=a^{2}+b^{2}-a b$. This domain will be denoted by $E$ and will be called the ring of Eisenstein integers. The units of $E$ are $\pm 1, \pm \omega$ and $\pm \bar{\omega}$. The primes of $E$ (up to a unit multiple) are the usual prime integers that are congruent to 2 modulo 3 and Eisenstein integers whose norm is a usual prime integer. Let $n$ be a natural number and let $\langle n\rangle$ be the principal ideal generated by $n$ in $E$. Then the factor ring 
$E /\langle n\rangle$ is isomorphic to the ring ${ }_{n}=\left\{a+b \omega \mid a, b \in \mathbb{Z}_{n}\right\}$, where $\mathbb{Z}_{n}$ is the ring of integers modulo n. Thus $E_{n}$ is a principal ideal ring. This ring is called the ring of Eisenstein integers modulo $n$. In [4] this ring is studied and its properties are investigated, its units are characterized and counted. It is easy to see that $a+b \omega$ is a unit in $E_{n}$ if and only if $N(a+b \omega)$ is a unit in $\mathbb{Z}_{n}$. Recall that a ring is local if it has a unique maximal ideal. It is shown that

(1) if $p$ is a prime integer, then the ring $E_{p^{k}}$ is local if and only if $p=3$ or $p \equiv 2(\bmod 3)$;

(2) let $\varphi(R)$ denote the number of units in a ring $R$, then $\varphi\left(E_{3^{k}}\right)=2 \times 3^{2 k-1}$ and

$$
\varphi\left(E_{p^{k}}\right)=\left\{\begin{array}{cccc}
p^{2 k-2}\left(p^{2}-1\right) & \text { if } & p \equiv 2 & (\bmod 3), \\
\left(p^{k}-p^{k-1}\right)^{2} & \text { if } & p \equiv 1 & (\bmod 3) .
\end{array}\right.
$$

In this article, some properties of the graphs $G\left(E_{n}\right)$ and $G_{E_{n}}$ are studied. The diameter, the girth, chromatic number and clique number, in terms of $n$, are found. Also, we prove that for each $n>1$, the graphs $G\left(E_{n}\right)$ and $G_{E_{n}}$ are Hamiltonian and the independence number of $G_{E_{n}}$ is calculated. An earlier study was carried out for the unit and unitary graphs for the ring of Gaussian integers modulo $n$, see [9].

Throughout the article, by a graph $G$ we mean a fnite undirected graph without loops or multiple edges. If the degree of each vertex in $G$ is equal to $k$, where $k$ is a positive integer, then $G$ is called $k$-regular graph. For a graph $G$ and for any two vertices $a$ and $b$ of $G$, we recall that a walk between $a$ and $b$ is an alternating sequence $a=v_{0}, e_{1}, v_{1}, e_{2}, \ldots, e_{k}, v_{k}=b$ of vertices and edges of $G$, denoted by

$$
a=v_{0} \stackrel{e_{1}}{\longrightarrow} v_{1} \stackrel{e_{2}}{\longrightarrow} \ldots \stackrel{e_{k}}{\longrightarrow} v_{k}=b,
$$

such that for every $i$ with $1 \leq i \leq k$, the edge $e_{i}$ has endpoints $v_{i-1}$ and $v_{i}$. Also, a path between $a$ and $b$ is a walk between $a$ and $b$ without repeated vertices. A cycle of a graph is a path such that the start and end vertices are the same. The number of edges (counting repeats) in a walk, path or a cycle, is called its length. A Hamiltonian path (cycle) in $G$ is a path (cycle) in $G$ that visits every vertex of $G$ exactly once. A graph is called Hamitonian if it contains a Hamiltonian cycle. For vertices $a$ and $b$ of $G$, we define $d(a, b)$ to be the length of a shortest path from $a$ to $b$ $(d(a, a)=0$ and $d(a, b)=\infty$ if there is no such path). The diameter of $G$ is

$$
\operatorname{diam}(G)=\sup \{d(a, b) \mid a, b \in V(G)\} .
$$

The girth of $G$, denoted by $\operatorname{gr}(G)$ is the length of a shortest cycle in $G,(\operatorname{gr}(G)=\infty$ if $G$ contains no cycle). For a positive integer $r$, a graph is called $r$-partite if the vertex set admits a partition into $r$ classes such that vertices in the same partition class are not adjacent. A $r$-partite graph is called complete if every two vertices in different parts are adjacent. The complete 2-partite graph (also called the complete bipartite graph) with exactly two partitions of size $n$ and $m$, is denoted by $K_{n, m}$. A complete graph on the $n$ vertices, denoted by $K_{n}$, is a graph such that every two of distinct vertices are adjacent. A clique in $G$ is a set of pairwise adjacent vertices of $G$. A clique of the maximum size is called a maximum clique. The clique number of $G$, denoted by $\omega(G)$, is the number of vertices of a maximum clique in $G$. We color the vertices of $G$ so that no two joined vertices have the same color. If we color the vertices, we call it a coloring of $G$. The chromatic number $\chi(G)$ of the graph $G$ is the minimum number of colors of colorings of $G$. The tensor product or Kronecker product $G \otimes H$ of two graphs $G$ and $H$ is the graph with vertex set $V(G) \times V(H)$, in which $(a, b)$ is adjacent to $(c, d)$ if and only if $a$ is adjacent to $c$ in $G$ and $b$ is adjacent to $d$ in $H$. For other notions not mentioned in this introduction, one can refer to [11, 15].

Throughout this article, the integers $p$ and $p_{i}$ are used implicitly to denote primes congruent to 2 modulo 3 , while $q$ and $q_{j}$ likewise denote prime integers congruent to 1 modulo 3 . For classical theorems and notations in commutative algebra, the interested reader is referred to [8]. 


\section{The Unit and Unitary Cayley Graphs of $E_{n}$}

In this section, we determine diameter and girth of the unit and unitary Cayley graphs of $E_{n}$. The case when $n$ is a power of a prime is considered first. Then the general case is considered.

\subsection{The Unit and Unitary Cayley Graphs of $E_{t^{n}}$}

For the sake of compeleteness, we mention here two important results that will be kept throghout the paper.

Proposition 1 [3, Proposition 2.2].

(a) Let $R$ be a ring. Then $G_{R}$ is a regular graph of degree $|U(R)|$.

(b) Let $R$ be a local ring with the maximal ideal $\underline{m}$. Then $G_{R}$ is a complete mutipartite graph whose partite sets are the cosets of $\underline{m}$ in $R$. In paticular, $G_{R}$ is a compelete graph if and only if $R$ is a field.

Theorem 1 [2, Theorem 3.1]. Let $R$ be a ring. Then $G(R)$ is a complete r-partite graph if and only if $R$ is a local ring with the maximal ideal $\underline{m}$ and $r=|R / \underline{m}|=2^{n}$, for some $n \in \mathbb{N}$ or $R$ is a finite field.

Theorem 2. Let $n$ be a positive integer. Then the following statements hold

(1) $\operatorname{diam}\left(G\left(E_{3^{n}}\right)\right)=\operatorname{diam}\left(G_{E_{3^{n}}}\right)=2$;

(2) $\operatorname{gr}\left(G\left(E_{3^{n}}\right)\right)=\operatorname{gr}\left(G_{E_{3^{n}}}\right)=3$.

P r o o f. For each positive integer $n, E_{3^{n}}$ is a local ring with the maximal ideal $\langle 2+\omega\rangle$, see [4]. Since $\varphi\left(E_{3^{n}}\right)=2 \times 3^{2 n-1}$, we have

$$
\left|\frac{E_{3^{n}}}{\langle 2+\omega\rangle}\right|=3
$$

Therefore, by Proposition 1, $G_{E_{3^{n}}}$ is a complete 3-partite graph and hence $\operatorname{diam}\left(G_{E_{3^{n}}}\right)=2$ and $\operatorname{gr}\left(G_{E_{3^{n}}}\right)=3$. Also, by Theorem 1, $G\left(E_{3^{n}} /\langle 2+\omega\rangle\right)$ is a complete bipartite graph. Thus $\operatorname{diam}\left(G\left(E_{3^{n}}\right)\right)=2$ and $\operatorname{gr}\left(G\left(E_{3^{n}}\right)\right)=3$.

Theorem 3. Let $n$ be a positive integer and $q$ be a prime integer congruent to 1 modulo 3. Then the following statements hold,

(1) $\operatorname{diam}\left(G\left(E_{q^{n}}\right)\right)=\operatorname{diam}\left(G_{E_{q^{n}}}\right)=2$;

(2) $\operatorname{gr}\left(G\left(E_{q^{n}}\right)\right)=\operatorname{gr}\left(G_{E_{q^{n}}}\right)=3$.

$\mathrm{P}$ r o o f. Since $q$ is a prime integer congruent to 1 modulo 3 , the ring $E_{q^{n}}$ is the product of the two local rings $E /\left\langle(a+b \omega)^{n}\right\rangle$ and $E /\left\langle(a+b \bar{\omega})^{n}\right\rangle$ that have the same number of elements. The ideals $\langle a+b \omega\rangle$ and $\langle a+b \bar{\omega}\rangle$ are the only maximal ideals of $E_{q^{n}}$, see [4]. Therefore, by [18, Theorem 3.5], we have

$$
\operatorname{diam}\left(G\left(E_{q^{n}}\right)\right)=\operatorname{diam}\left(G_{E_{q^{n}}}\right)=2 .
$$

On the other hand, in view of the proof of [7, Proposition 5.10] and [3, Theorem 3.2], we obtain

$$
\operatorname{gr}\left(G\left(E_{q^{n}}\right)\right)=\operatorname{gr}\left(G_{E_{3^{n}}}\right)=3 .
$$


Lemma 1 [19, Lemma 4.1]. Let $R$ be a finite ring and $a \in R$. The following statements are equivalent:

(1) $a \in J(R)$;

(2) $a+u \in U(R)$ for any $u \in U(R)$.

Theorem 4 [9, Theorem 2.6]. Let $R \cong R_{1} \times R_{2} \times \ldots \times R_{n}$ be a finite ring, where $\left(R_{i}, \underline{m}_{i}\right)$ is a local ring, for each $i=1, \cdots, n$. Then the following statements are equivalent:

(1) $2 \in J(R)$;

(2) $G_{R}=G(R)$;

(3) for every $i=1, \ldots, n,\left|R_{i}\right|$ is even.

Theorem 5. Let $n$ be a positive integer and $p$ be a prime integer congruent to 2 modulo 3 . Then the following statements hold

(1) $\operatorname{diam}\left(G_{E_{p^{n}}}\right)= \begin{cases}1 & \text { if } n=1 \\ 2 & \text { if } n>1\end{cases}$

(2) $\operatorname{diam}\left(G\left(E_{p^{n}}\right)\right)=2$;

(3) $\operatorname{gr}\left(G\left(E_{p^{n}}\right)\right)=\operatorname{gr}\left(G_{E_{p^{n}}}\right)=3$.

$\mathrm{P}$ r o o f. Since $p$ is a prime integer congruent to 2 modulo $3, p$ is an Eisenstein prime integer. Hence $E_{p}$ is a field. If $n>1$, then the ring $E_{p^{n}}$ is a local ring with the maximal ideal $\langle p\rangle$. Since

$$
\varphi\left(E_{p^{n}}\right)=p^{2 k-2}\left(p^{2}-1\right),
$$

we obtain that (see [4]):

$$
\left|\frac{E_{p^{n}}}{\langle p\rangle}\right|=p^{2}
$$

If $p=2$, then it follows from Theorem 4 that $G\left(E_{p^{n}}\right)=G_{E_{p^{n}}}$. In this case, by [3, Theorem 3.1] and [3, Theorem 3.2], we obtain $\operatorname{gr}\left(G\left(E_{p^{n}}\right)\right)=\operatorname{gr}\left(G_{E_{p^{n}}}\right)=3$ and

$$
\operatorname{diam}\left(G\left(E_{p^{n}}\right)\right)=\operatorname{diam}\left(G_{E_{p^{n}}}\right)=\left\{\begin{array}{lll}
1 & \text { if } & n=1 \\
2 & \text { if } & n>1
\end{array}\right.
$$

We now assume that $p \neq 2$. Then $G_{E_{p^{n}}}$ is a complete $p^{2}$-partite graph. Therefore, $\operatorname{diam}\left(G_{E_{p^{n}}}\right)=2$ and $\operatorname{gr}\left(G_{E_{p^{n}}}\right)=3$. Since $G\left(E_{p^{n}} /\langle p\rangle\right)$ is a complete $\left(p^{2}+1\right) / 2$-partite graph, we obtain that

$$
\operatorname{diam}\left(G\left(E_{p^{n}}\right)\right)=2, \quad \operatorname{gr}\left(G\left(E_{p^{n}}\right)\right)=3 .
$$




\subsection{Diameter and Girth for the Graphs $G_{E_{n}}$ and $G\left(E_{n}\right)$}

The general case is now investigated. It is well known [8, Theorem 8.7] that every finite commutative ring can be expressed as a direct product of finite local rings, and this decomposition is unique up to permutations of such local rings. Throughout this section we assume that $R \cong R_{1} \times R_{2} \times \ldots \times R_{t}$ is a finite commutative ring, where each $R_{i}$ is a finite commutative local ring with the maximal ideal $\underline{m}_{i}$. Since $\left(u_{1}, \ldots, u_{t}\right)$ is a unit of $R$ if and only if each $u_{i}$ is a unit in $R_{i}$, we see immediately that $G_{R} \cong G_{R_{1}} \otimes G_{R_{2}} \ldots \otimes G_{R_{t}}$ and $G(R) \cong G\left(R_{1}\right) \otimes G\left(R_{2}\right) \ldots \otimes G\left(R_{t}\right)$. We denote by $K_{i}$ the (finite) residue field $R_{i} / \underline{m}_{i}$ and $f_{i}=\left|K_{i}\right|$. We also assume (after appropriate permutation of factors) that $f_{1} \leq f_{2} \leq \ldots \leq f_{t}$.

Theorem 6. Let $n>1$ be an integer with at least two distinct prime factors. Then

$$
\operatorname{diam}\left(G_{E_{n}}\right)=\operatorname{diam}\left(G\left(E_{n}\right)\right)=2 .
$$

P r o o f. Let

$$
n=3^{k} \times \prod_{i=1}^{m} p_{i}^{\alpha_{i}} \times \prod_{j=1}^{l} q_{j}^{\beta_{j}},
$$

where $p_{i}$ and $q_{j}$ are prime integers such that $p_{i} \equiv 2(\bmod 3)$ and $q_{j} \equiv 1(\bmod 3)$, then

$$
E_{n} \cong E_{3^{k}} \times \prod_{i=1}^{m} E_{p_{i}^{\alpha_{i}}} \times \prod_{j=1}^{l} E_{q_{j}^{\beta_{j}}},
$$

see [4]. This shows that, $E_{n}$ is isomorphic to a direct product of finite local rings $\left(R_{i}, \underline{m}_{i}\right)$, such that for every $i,\left|R_{i} / \underline{m}_{i}\right|=3$ or $p_{i}^{2}$ or $q_{j}$. By [3, Theorem 3.5 (b)], we conclude that

$$
\operatorname{diam}\left(G_{E_{n}}\right)=\operatorname{diam}\left(G\left(E_{n}\right)=2 .\right.
$$

Theorem 7. Let $n>1$ be an integer with at least two distinct prime factors. Then

$$
\operatorname{gr}\left(G_{E_{n}}\right)=\operatorname{gr}\left(G\left(E_{n}\right)\right)=3 .
$$

P r o o f. By the argument similar to that above, we conclude that

$$
E_{n} \cong E_{3^{k}} \times \prod_{i=1}^{m} E_{p_{i}^{\alpha_{i}}} \times \prod_{j=1}^{l} E_{q_{j}^{\beta_{j}}} .
$$

Thus, by [3, Theorem 3.2], we obtain $\operatorname{gr}\left(G_{E_{n}}\right)=3$. On the other hand, in view of the proof of [7, Theorem 5.10], we have $\operatorname{gr}\left(G\left(E_{n}\right)\right) \in\{3,4\}$.

Since $n$ is an integer with at least two distinct prime factors, we can assume that $n=a b$ with $\operatorname{gcd}(a, b)=1$. It is clear that

$$
\operatorname{gcd}\left(a^{2}+b^{2}-a b, n\right)=1 .
$$

Thus, $N(a+b \omega)$ is a unit in $\mathbb{Z}_{n}$, and so $a+b \omega$ is a unit in $E_{n}$. This showes that $x=a$ and $y=b \omega$ are adjacent. Now, by taking $z=b+a \omega$, we have $x+z=(a+b)+a \omega$ and $y+z=b+(a+b) \omega$. Clearly, $N(x+z)=N(y+z)=a^{2}+b^{2}+a b$ is a unit in $\mathbb{Z}_{n}$ which implies that $x+z$ and $y+z$ are unit elements of $E_{n}$. Therefore, we obtain the cycle

$$
x \longrightarrow y \longrightarrow z \longrightarrow x .
$$

This implies that $\operatorname{gr}\left(G\left(E_{n}\right)\right)=3$. 


\subsection{Some Graph Invariants of Graphs $G_{E_{n}}$ and $G\left(E_{n}\right)$}

In the sequel, we obtain the clique number and the chromatic number for the graphs $G_{E_{n}}$ and $G\left(E_{n}\right)$.

Theorem 8. Let $n>1$ be an integer and

$$
n=3^{k} \times \prod_{i=1}^{m} p_{i}^{\alpha_{i}} \times \prod_{j=1}^{l} q_{j}^{\beta_{j}} .
$$

Then the following statements hold

(1) if $3 \mid n$, then $\chi\left(G_{E_{n}}\right)=\omega\left(G_{E_{n}}\right)=3$ and $\alpha\left(G_{E_{n}}\right)=n^{2} / 3$;

(2) if $3 \nmid n$, then

$$
\chi\left(G_{E_{n}}\right)=\omega\left(G_{E_{n}}\right)=\min \left\{p_{i}^{2}, q_{j}\left|1 \leq i \leq m, 1 \leq j \leq l, p_{i}\right| n, q_{j} \mid n\right\}
$$

and

$$
\alpha\left(G_{E_{n}}\right)=\frac{n^{2}}{\min \left\{p_{i}^{2}, q_{j}\left|1 \leq i \leq m, 1 \leq j \leq l, p_{i}\right| n, q_{j} \mid n\right\}} .
$$

P r o o f. 1 . Let $k$ be the biggest positive integer such that $3^{k} \mid n$. Then

$$
E_{n}=E_{3^{k}} \times \prod_{i=1}^{m} E_{p_{i} \alpha_{i}} \times \prod_{j=1}^{l} E_{q_{j}^{\beta_{j}}} .
$$

Since $E_{3^{k}}$ is a local ring with the maximal ideal $\langle 2+\omega\rangle$ and

$$
\left|\frac{E_{3^{k}}}{\langle 2+\omega\rangle}\right|=3
$$

it follows from [3, Proposition 6.1] that $\chi\left(G_{E_{n}}\right)=\omega\left(G_{E_{n}}\right)=3$ and $\alpha\left(G_{E_{n}}\right)=n^{2} / 3$.

2. If $3 \nmid n$, then it yields that $E_{n}$ is isomorphic to a direct product of finite local rings $\left(R_{i}, \underline{m}_{i}\right)$, such that for every $i,\left|R_{i} / \underline{m}_{i}\right|=p_{i}^{2}$ or $q_{j}$. Thus by [3, Proposition 6.1], we have

$$
\chi\left(G_{E_{n}}\right)=\omega\left(G_{E_{n}}\right)=k=\min \left\{p_{i}^{2}, q_{j} \mid 1 \leq i \leq m, 1 \leq j \leq l\right\}
$$

and $\alpha\left(G_{E_{n}}\right)=n^{2} / k$.

Theorem 9. Let $n>1$ be an integer and

$$
n=3^{k} \times \prod_{i=1}^{m} p_{i}^{\alpha_{i}} \times \prod_{j=1}^{l} q_{j}^{\beta_{j}} .
$$

Then the following statements hold

(1) if $2 \mid n$, then $\chi\left(G\left(E_{n}\right)\right)=\omega\left(G\left(E_{n}\right)\right)=4$;

(2) if $2 \nmid n$, then

$$
\chi\left(G\left(E_{n}\right)\right)=\omega\left(G\left(E_{n}\right)\right)=\frac{1}{2^{1+m+l}} \times \prod_{i=1}^{m}\left(p_{i}^{2 \alpha_{i}}-p_{i}^{2 \alpha_{i}-2}\right) \times \prod_{j=1}^{l}\left(q_{j}^{\beta_{j}}-q_{j}^{\beta_{j}-1}\right)^{2}+m+2 l+1 .
$$


P r o o f. Since $n=3^{k} \times \prod_{i=1}^{m} p_{i}^{\alpha_{i}} \times \prod_{j=1}^{l} q_{j}^{\beta_{j}}$, we have

$$
E_{n}=E_{3^{k}} \times \prod_{i=1}^{m} E_{p_{i}^{\alpha_{i}}} \times \prod_{j=1}^{l} E_{q_{j}} .
$$

1. If $2 \mid n$, then $2 \notin U\left(E_{n}\right)$. Hence, in view of the proof of [21, Theorem 2.2], we have

$$
\chi\left(G\left(E_{n}\right)\right)=\omega\left(G\left(E_{n}\right)\right)=4 .
$$

2. If $2 \nmid n$, Then $2 \in U\left(E_{n}\right)$. By an argument similar to that above, we conclude that

$$
\chi\left(G\left(E_{n}\right)\right)=\omega\left(G\left(E_{n}\right)\right)=\frac{1}{2^{1+m+l}} \times \prod_{i=1}^{m}\left(p_{i}^{2 \alpha_{i}}-p_{i}^{2 \alpha_{i}-2}\right) \times \prod_{j=1}^{l}\left(q_{j}^{\beta_{j}}-q_{j}^{\beta_{j}-1}\right)^{2}+m+2 l+1 .
$$

We now state our final result.

Theorem 10. For each integer $n>1$, the graphs $G\left(E_{n}\right)$ and $G_{E_{n}}$ are Hamitonian.

P r o o f. Let $n>1$ be an integer. By Theorem 2, Theorem 3, Theorem 5 and Theorem 6 , the graphs $G\left(E_{n}\right)$ and $G_{E_{n}}$ are connected. Thus $G\left(E_{n}\right)$ is Hamiltonian graph, by [22, Theorem 2.1]. Also, it follows from [20, Lemma 4] that $G_{E_{n}}$ is Hamiltonian graph.

\section{Concluding Remarks}

In this article, the diameter, the girth, the chromatic number and the clique number of $G\left(E_{n}\right)$ and $G_{E_{n}}$ are studied. We also prove that for each $n>1$, the graphs $G\left(E_{n}\right)$ and $G_{E_{n}}$ are Hamiltonian and the independence number of $G_{E_{n}}$ is calculated. We end our paper with the following two open questions:

Question 1. Is there any closed formula for $\alpha\left(G\left(E_{n}\right)\right)$ ?

Question 2. When are $G\left(E_{n}\right)$ and $G_{E_{n}}$ Eulerian?

\section{Acknowledgments}

I would like to thank the referee for the valuable suggestions and comments.

\section{REFERENCES}

1. Aalipour G., Akbari S. On the Cayley graph of a commutative ring with respect to its zero-divisors. Comm. Algebra, 2016. Vol. 44, No. 4. P. 1443-1459.

2. Akbari S., Estaji E., Khorsandi M.R. On the unit graph of a non-commutative ring. Algebra Colloq., 2015. Vol. 22. P. 817-822. DOI: 10.1142/S100538671500070X

3. Akhtar R., Jackson-Henderson T., Karpman R., Boggess M., Jiménez I., Kinzel A., Pritikin D. On the unitary Cayley graph of a finite ring. Electron. J. Combin., 2009. Vol. 16, No. 1. Art. no. R117. DOI: $10.37236 / 206$

4. Alkam O., Abu Osba E. On Eisenstein integers modulo n. Int. Math. Forum., 2010. Vol. 5, No. 22. P. $1075-1082$.

5. Anderson D. F., Badawi A. The total graph of a commutative ring. J. Algebra, 2008. Vol. 320. No. 7. P. 2706-2719. DOI: 10.1016/j.jalgebra.2008.06.028 
6. Anderson D. F., Livingston P. S. The zero-divisor graph of a commutative ring. J. Algebra, 1999. Vol. 217, No. 2. P. 434-447. DOI: 10.1006/jabr.1998.7840

7. Ashrafi N., Maimani H.R., Pournaki M.R., Yassemi S. Unit graphs associated with rings. Comm. Algebra, 2010 Vol. 38. P. 2851-2871. DOI: 10.1080/00927870903095574

8. Atiyah M. F., MacDonald I. G. Introduction to Commutative Algebra. Menlo Park, California, London, Don Mills, Ontario: Addison-Wesley Publishing Co., 1969. 128 p.

9. Bahrami A., Jahani-Nezhad R. Unit and unitary Cayley graphs for the ring of Gaussian integers modulo n. Quasigroups Related Systems, 2017. Vol. 25, No. 2. P. 189-200.

10. Beck I. Coloring of commutative rings. J. Algebra, 1988 Vol. 116. No. 1. P. $208-226$. DOI: 10.1016/0021-8693(88)90202-5

11. Bondy J.A., Murty U.S.R. Graph Theory with Applications. New York, Amsterdam, Oxford: NorthHolland, 1976. 264 p.

12. Cayley A. Desiderata and Suggestions: No. 2. The Theory of Groups: Graphical Representation. Amer. J. Math., 1878. Vol. 1, No. 2. P. 174-176.

13. Chung F. R. K. Diameters and eigenvalues. J. Amer. Math. Soc., 1989. Vol. 2, No. 2. P. $187-196$. DOI: $10.2307 / 1990973$

14. Dejter I. J., Giudici R. E. On unitary Cayley graphs. J. Combin. Math. Comput., 1995. Vol. 18. P. 121124.

15. Diestel R. Graph Theory. Berlin, Heidelberg: $\quad$ Springer-Verlag, 2001.428 p. DOI: $10.1007 / 978-3-662-53622-3$

16. Grimaldi R. P. Graphs from rings. Congr. Numer., 1990. Vol. 17. P. 95-103.

17. Ireland K., Rosen M. A Classical Introduction to Modern Number Theory. NY: Springer-Verlag, 1990. 394 p. DOI: $10.1007 / 978-1-4757-2103-4$

18. Khashyarmanesh K., Khorsandi M.R. A generalization of the unit and unitary Cayley graphs of a commutative ring. Acta Math. Hungar., 2012. Vol. 137. P. 242-253. DOI: 10.1007/s10474-012-0224-5

19. Kiani D., Aghaei M. M. H. On the unitary Cayley graph of a ring. Electron. J. Combin., 2012. Vol. 19, No. 2. Art. no. P10. DOI: $10.37236 / 2214$

20. Lanski C., Maróti A. Ring elements as sums of units. Cent. Eur. J. Math., 2009. Vol. 7. P. $395-399$. DOI: $10.2478 / \mathrm{s} 11533-009-0024-5$

21. Maimani H. R., Pournaki M.R., Yassemi S. Weakly perfect graphs arising from rings. Glasg. Math. J., 2010. Vol. 52, No. 3. P. 417-425. DOI: 10.1017/S0017089510000108

22. Maimani H.R., Pournaki M.R., Yassemi S. Necessary and sufficient conditions for unit graphs to be Hamiltonian. Pacific J. Math., 2011. Vol. 249, No. 2. P. 419-429. 